\title{
A 3D UNSTEADY NUMERICAL SIMULATION OF THE REFRIGER- ANT FLOW EVAPORATION IN A PLATE HEAT EXCHANGER
}

\author{
Austrian Institute of Technology \\ Giefinggasse 2, 1210 Vienna, Austria \\ mirza.popovac@ait.ac.at
}

Mirza Popovac, Gerwin Schmid and Michael Lauermann

Keywords: Multi-phase, pseudo-fluid, phase-change, CoolProp, OpenFoam, CFD.

\begin{abstract}
This work presents a robust CFD approach for simulating the refrigerant flow evaporation within a plate heat exchanger of practical relevance with respect to the geometry and operating conditions. The purpose of this work is to assess the applicability of the numerical fluid flow simulations in predicting the thermal behavior and the fluid flow performance (including the phase change) of a plate heat exchanger with a realistic geometry and under realistic operating conditions. To that purpose, the basic structure of the CFD solver necessary for the numerical simulation of the occurring fluid flow phenomena is discussed here, together with the corresponding boundary conditions, fluid properties treatment, and related geometry issues. The results obtained for a specific plate heat exchanger are validated against experimental results found in available literature.
\end{abstract}




\section{INTRODUCTION}

One of the key components in various thermal machines are plate heat exchangers, which have the purpose to transfer the heat from a hot to a cold fluid. Typically it is expected that these heat exchangers exhibit the highest possible heat transfer coefficient with lowest possible hydraulic losses. The present paper focuses on a numerical method for analyzing the thermal behavior of a plate heat exchanger with refrigerant flow evaporation: while flowing through the plate channels of a heat exchanger, the refrigerant is evaporating and simultaneously cooling down the liquid (in this case the water) on the opposite side of the plate. The aim of the present work is to assess the applicability of this numerical approach, based on the computational fluid dynamics (CFD), to analyze the performance of plate heat exchangers of realistic geometries and under realistic operating conditions.

A great challenge in this work comes from the requirement for a realistic representation of the geometric features of the plate channels within a single layer of analyzed plate heat exchanger. On the one hand there is a huge variation in the characteristic length scales (high length to height ratio of the plate layer), and on the other there is an interlacing wavy geometry of the plate channels. Both of these aspects are setting high demands on the meshing, and as a result sufficiently high mesh quality cannot always be assured.

The most critical part of the present work, however, is the modeling of analyzed two-phase phenomena. Given the complexity of the desired application, in this paper the focus is on the robustness of the physical description of the problem under consideration, and the corresponding stability of the numerical simulation. Therefore, in the selected approach the two nonmiscible phases (refrigerant liquid and vapor phase) are treated as a single fluid with combined transport properties. The properties of individual phases are obtained from the CoolProp simulation platform for solving fluid equations of state and transport properties [1]. For thus defined pseudo-fluid, the governing equations for continuity, momentum and energy conservation are solved [2]. The phase-change process is described through the transfer function, which represents the volume fraction of a single phase. The assumption being introduced here is to express this function explicitly through the local temperature (comparing it with the saturation temperature), and implicitly include the related phase-change effects through the physical properties of the involved phases (e.g. the latent heat). The abovementioned approach significantly improves the numerical performance of the simulation method, in particular when the numerical mesh quality and the computational time requirements are concerned.

The numerical results, obtained using $O$ penFOAM ${ }^{\circledR}$ library suite [3], are validated against the available experimental results found in literature. The preliminary results using the adopted approach are showing that the basic flow pattern and the phase distribution within the plate heat exchanger channels can be identified. Comparing the results of the experiments and simulations, which are quantified through the integral values of momentum and heat transfer, the overall agreement is satisfactory.

\section{HEAT EXCHANGER GEOMETRY}

The creation of the plate heat exchanger geometry is very challenging task, and the meshing of this geometry is even more demanding. Nevertheless, this is the starting point for a numerical simulation, and it is a critical element when the quality of the simulation results is concerned. For a realistic representation of the geometric features of a single layer of the heat exchanger under investigation, SolidWorks ${ }^{\mathrm{TM}}$ package has been used to create a parametric model with characteristic width, length and chevron angle. 
Shown in Figure 1 is the plate heat exchanger model of Huang et al. [4], which has been considered in the present analysis. Due to its highly complex geometry, only one layer of this heat exchanger has been included in the analysis: this layer consists of the channels through which the refrigerant is flowing, while the influence of the water on the secondary side has accounted for through the appropriate boundary condition. In this calculation the incoming and outgoing zones of the analyzed layer (yellow) were slightly modified with respect to the real geometry: there is no dedicated manifold distributor near the inlet or outlet of the heat exchanger, but rather the same geometry as throughout the whole plate (same channel size and chevron angle). Through the inlet (blue) and outlet (green) sections the operating conditions have been imposed. The remaining part (red) represents the walls through which the heat transfer with the secondary fluid (water) is taking place.
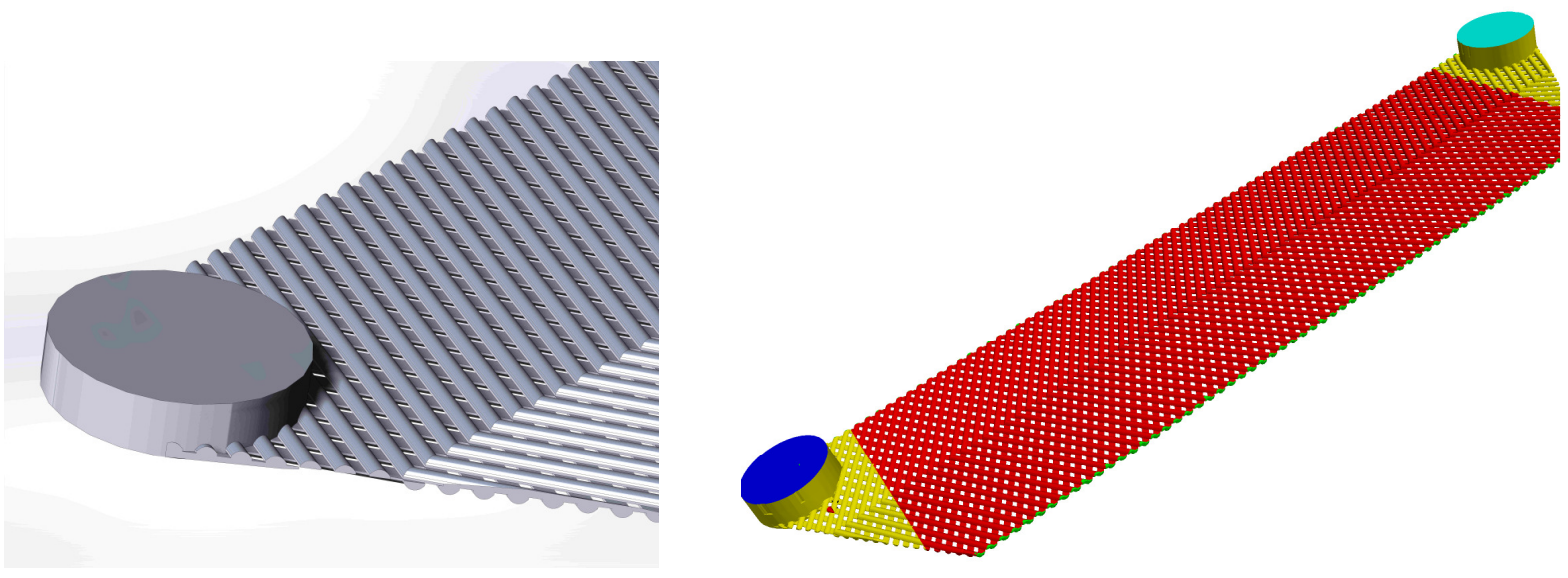

Figure 1: plate heat exchanger geometry detail (left), and the numerical mesh (right).

Using the geometry created as explained above, the numerical mesh has been created using SnappyHexMesh tool from the OpenFoam suite. For the given complexity of the simulated geometry, the only feasible meshing process is to use this automatic meshing approach, although its immanent feature is that they cannot assure the overall mesh quality (and the mesh quality will influence the stability and quality of the numerical simulation - especially in the case of the multi-phase flow simulations). One possibility of improving the mesh quality is to go for higher refinement levels (finer mesh), but that implies larger meshes (number of cells) and longer simulation times.

\section{NUMERICAL FLUID FLOW SOLVER}

For the present numerical analysis the open-source package OpenFoam has been used: it is based on the finite-volume method, and owing to its object-oriented structure it enables straightforward implementations of the necessary improvements (in this case, a realistic treatment of refrigerant properties, and the flow evaporation model). For the simulation of the refrigerant flow evaporation within a plate heat exchanger, the transient flow solver has been selected, based on the combined PISO-SIMPLE method.

The problem under consideration involves two nonmiscible phases of a single constituent: refrigerant liquid and vapor phase. In the adopted approach, these two phases are treated as a pseudo-fluid (with combined fluid properties), for which the time scales of the transport between the phases is much smaller than the integral time scale of the flow. This assumption 
invokes the thermodynamic equilibrium requirement, in which case it is shown that the twophase flow obeys the governing laws of a single-phase flow [5]. In other words, the flow is described with the equations for conservation of mass, momentum and enthalpy, as given below:

$$
\begin{gathered}
\frac{\partial \rho}{\partial t}+\frac{\partial \rho u_{j}}{\partial x_{j}}=0 \\
\frac{\partial \rho u_{i}}{\partial t}+\frac{\partial \rho u_{j} u_{i}}{\partial x_{j}}=\frac{\partial}{\partial x_{j}}\left[\left(\mu+\mu_{t}\right) \frac{\partial u_{i}}{\partial x_{j}}\right]-\frac{\partial p}{\partial x_{i}} \\
\frac{\partial \rho h}{\partial t}+\frac{\partial \rho u_{j} h}{\partial x_{j}}=\frac{\partial}{\partial x_{j}}\left[\left(\frac{\lambda}{c_{p}}+\frac{\lambda_{t}}{c_{p}}\right) \frac{\partial h}{\partial x_{j}}\right]
\end{gathered}
$$

The enthalpy approach is a conservative approach which is valid in a general phase-change case [6]. The enthalpy includes both the latent heat $H$ and the sensible heat (measured by the temperature $T$ ): $h=c_{p} T+H$, whereby the latent heat $H$ is being treated explicitly during the phase-change process. The combined properties of pseudo-fluid (density $\rho$, viscosity $\mu$, specific heat capacity $c_{p}$, thermal conductivity $\lambda$ ) are defined as a weight mixture of the individual fluid properties: $\phi=\phi_{\text {vapor }} \alpha_{\text {vapor }}+\phi_{\text {liquid }} \alpha_{\text {liquid }}\left(\phi=\rho, c_{p}, \mu, \lambda\right)$. The weighting function is represented by the volume fraction of a single phase $\alpha$, with $\alpha_{v a p o r}=1-\alpha_{\text {liquid }}$.

The effects of turbulence are introduced through the eddy viscosity $\mu_{t}$, here defined using the $k-\mathcal{E}$ model [7] with the realizability constrain imposed through the time scale $T_{r}$ :

$$
\begin{gathered}
\mu_{t}=\rho c_{\mu} k T_{r} \\
\frac{\partial \rho k}{\partial t}+\frac{\partial \rho u_{j} k}{\partial x_{j}}=\frac{\partial}{\partial x_{j}}\left[\left(\mu+\frac{\mu_{t}}{\sigma_{k}}\right) \frac{\partial k}{\partial x_{j}}\right]+P_{k}-\rho \varepsilon \\
\frac{\partial \rho \varepsilon}{\partial t}+\frac{\partial \rho u_{j} \varepsilon}{\partial x_{j}}=\frac{\partial}{\partial x_{j}}\left[\left(\mu+\frac{\mu_{t}}{\sigma \varepsilon}\right) \frac{\partial \varepsilon}{\partial x_{j}}\right]+\frac{C_{1} P_{k}-C_{2} \rho \varepsilon}{T_{r}} \\
T_{r}=\max \left[\frac{k}{\varepsilon}, C_{\tau}\left(\frac{v}{\varepsilon}\right)^{1 / 2}\right]
\end{gathered}
$$

\subsection{Flow evaporation model}

Given the complexity of the phase-change flow physics, its modeling represents a critical element in the numerical simulation of the plate heat exchanger with flow evaporation: it needs to be an appropriate phase-change model which will sufficiently capture the main flow phenomena, and yet be sufficiently fast and flexible in order to be suitable for an industrial application analyzed in this work. Furthermore, for the flow type under consideration here, there is the stability criterion (expressed through the Courant number) which correlates allowed spatial and temporal discretization characteristics (i.e. the time step and the mesh size). In the case of plate heat exchangers, however, the mesh quality is typically rather low. As a consequence, not only is the quality of the results reduced, but also the numerical simulation can get unstable or prohibitively long. 
Aiming at the balance between the accuracy and applicability, a phenomenological approach for the phase-change model has been pursued, where the phase-change process is strictly controlled by the temperature. Namely, the phase kinetic is implemented by prescribing the phase fraction as the function of temperature: the pseudo-fluid is in its liquid phase if the local temperature is below the saturation temperature, and inversely it is in its vapor phase when the local temperature is above the saturation temperature. Corollary to this assumption is that the robustness of the model is significantly increased, while the main phase-change features are accounted for: the temperature is its dominant characteristic, and the saturation temperature is dynamically determined from the property table based on the local flow pressure. As the model parameter, the linear temperature range in which the change between the phases is taking place, can be extracted from the Clausius-Clapeyron relation [5].

\subsection{Fluid properties}

As the focus in this work is on the flows of refrigerants, in order to capture the totality of the fluid flow phenomena within a heat exchanger with flow evaporation, it is necessary to account for a realistic variation of the relevant fluid properties: density, viscosity, heat conductivity and specific heat capacity. To that purpose, the volumetric fields for fluid properties have been implemented into the simulation solver, and they are being fed from the external property tables by interpolating respective values based on the local flow conditions (pressure and temperature). These property tables are generated using simulation tools for fluid equations of state and transport properties, and in the present case the open-source software CoolProp has been used.

\subsection{Boundary conditions}

The inlet is defined by the fixed values (Dirichlet boundary condition type) for the velocity, temperature (enthalpy) and turbulent quantities, and the zero gradient (Neumann boundary condition type) for the pressure. The imposed velocity is obtained from the mass flux specified by the operating conditions, together with the related temperature, whereas the turbulent quantities at the inlet are obtained from the required turbulent intensity. The pressure at the outlet is defined by the fixed value specified by the operating conditions, and for the velocity, temperature and the turbulent quantities the zero gradient is specified.

The heat transfer through the plate walls is defined by the Robin type boundary condition: there the temperature of the refrigerant in the near-wall region within the plate channel is dependent on the heat transfer coefficient and the temperature of the secondary fluid at the opposite side of the plate wall. In this way the convective heat transfer from the secondary side (water) is accounted for, together with the 1D assumption for the conduction through the wall of the plate heat exchanger.

\section{RESULTS}

In order to assess the ability of the proposed simulation approach in predicting the thermal behavior and the fluid flow performance of a plate heat exchanger, the comparison between the numerical investigations and the experimental results has been performed. Selected for the comparison is the heat exchanger geometry of Huang et al. [4], with the chevron angle of $60^{\circ}$. For the quantification of the fluid flow and heat transfer characteristics, a series of numerical simulations has been performed for two refrigerants (R507a and R134a) at two operating conditions at approximately the same saturation temperature levels. 
Summarized in the Table 1 are the boundary conditions applied for four different heat exchanger simulations. From the inlet mass flux, the imposed inlet velocity of the refrigerant has been recalculated for the high and low flow rates respectively. The imposed pressure at the outlet was selected such that for both refrigerants the saturation temperatures are approximately the same. On the thermal side of the secondary fluid, the inlet and outlet temperatures of the water are prescribed, and together with a specified mass flow rate the cooling rate is defined. This, in turn, gives the outlet temperature of the refrigerant, for the specified refrigerant inlet temperature.

\begin{tabular}{|c|c|c|c|c|c|}
\hline & & $\begin{array}{c}\text { R507a } \\
\text { high flow rate }\end{array}$ & $\begin{array}{c}\text { R507a } \\
\text { low flow rate }\end{array}$ & $\begin{array}{c}\text { R134a } \\
\text { high flow rate }\end{array}$ & $\begin{array}{c}\mathrm{R} 134 \mathrm{a} \\
\text { low flow rate }\end{array}$ \\
\hline \multirow{3}{*}{ 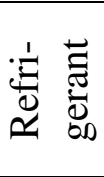 } & $V_{\text {in }}[\mathrm{m} / \mathrm{s}]$ & 0.03 & 0.02 & 0.03 & 0.02 \\
\hline & $P_{\text {in }}[k P a]$ & 756.3 & 749.1 & 324.3 & 315.6 \\
\hline & $P_{\text {out }}[k P a]$ & 742.4 & 742.8 & 306.7 & 307.2 \\
\hline \multirow{3}{*}{$\begin{array}{l}\dot{\bar{d}} \\
\dot{z} \\
3\end{array}$} & $T_{\text {in }}\left[{ }^{o} C\right]$ & 16.3 & 16.3 & 15.2 & 14.5 \\
\hline & $T_{\text {out }}\left[{ }^{o} \mathrm{C}\right]$ & 12 & 12.6 & 11.2 & 11.5 \\
\hline & $Q_{\text {cool }}[k W]$ & 14.4 & 12.2 & 13.3 & 10.1 \\
\hline
\end{tabular}

Table 1: operating conditions imposed as the boundary conditions.

The basic flow pattern can be seen in the Figure 2, which shows the velocity magnitude distribution of the refrigerant within the plate heat exchanger channels. The main conclusion from the obtained flow pattern is that good uniformity of the refrigerant flow has been achieved, which is an important ingredient for a good thermal behavior of the heat exchanger. The flow uniformity is the consequence of very complex surface structure, which distributes evenly the flow already in the entering section. However, such hydrodynamic behavior comes with the price, expressed in the overall pressure drop along the plate heat exchanger. The temperature distribution follows the main flow pattern: in the narrow parts of the plate channels (where two plates are interlacing) the velocity is increasing, carrying away the heat more effectively and hence locally reducing the refrigerant temperature. On the other hand, in larger channel pockets the refrigerant velocity is decreasing, and its temperature is increasing.
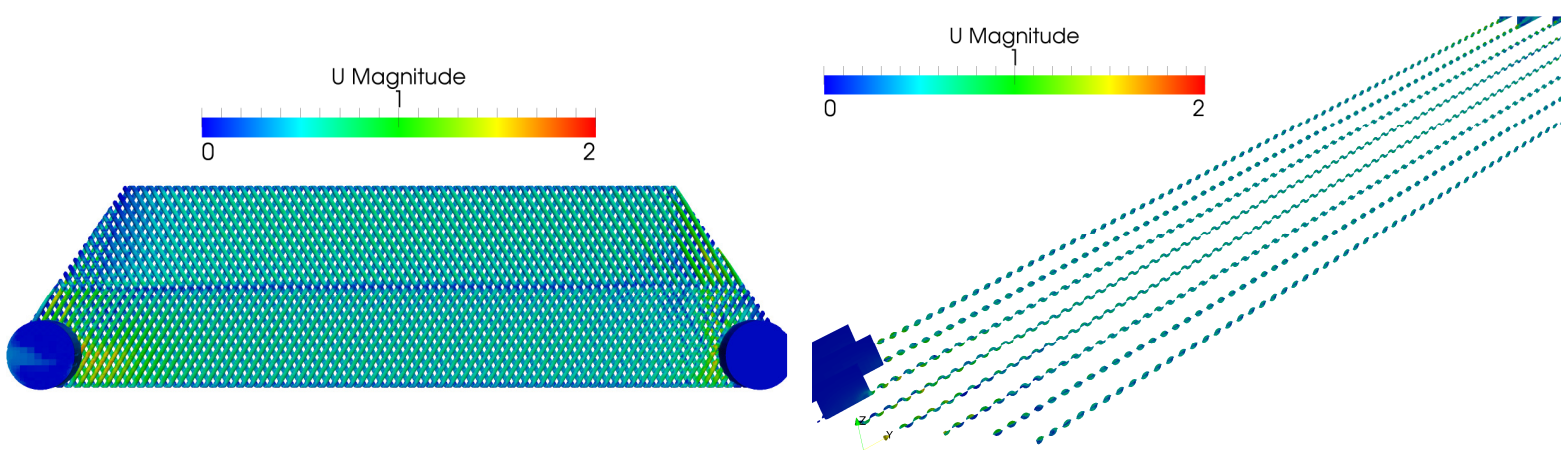

Figure 2: snapshot of the velocity distribution within the plate heat exchanger.

It is stipulated by the adopted phase-change model, that the phase distribution is dictated by the temperature distribution: the pockets of lower temperature evaporate the last, during the flow time. The overall characterization of the refrigerant flow evaporation, however, can be expressed through the domain integrated values of the phases. This has been shown in Figure 3, where the temporal evolution of the volume averaged liquid phase within the domain 
(green line) indicates the characteristic time of analyzed plate heat exchanger needed for reaching its steady-state operation. The same conclusion can be drawn from the surface averaged temperature at the heat exchanger's outflow (red line).

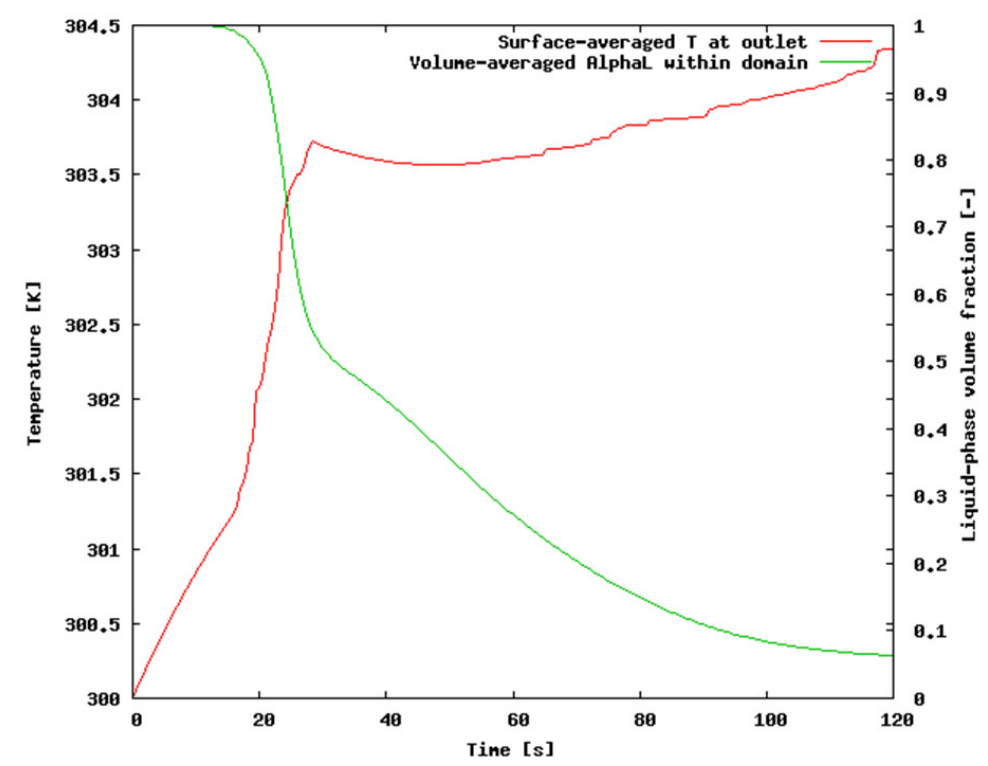

Figure 3: volume-averaged liquid phase, surface-averaged temperature.

From the data given in the Table 2 one can see that the prediction of the thermal characteristics is somewhat more difficult than the hydrodynamic characteristics. Namely, looking at the pressure drop, there is approximately $21 \%$ difference between the experimental and numerical results (in the average), whereas the difference in the heat transfer coefficient is approximately $27 \%$ (in the average). Nevertheless, even for thus obtained results a satisfactory agreement can be considered, because in all investigated cases there is a correct trend when changing the operating conditions: this systematic discrepancy can be reduced by certain finetuning of the model, which is subject of the future work.

\begin{tabular}{|c|c|c|c|c|c|}
\hline & & $\begin{array}{c}\text { R507a } \\
\text { high flow rate }\end{array}$ & $\begin{array}{c}\text { R507a } \\
\text { low flow rate }\end{array}$ & $\begin{array}{c}\text { R134a } \\
\text { high flow rate }\end{array}$ & $\begin{array}{c}\text { R134a } \\
\text { low flow rate }\end{array}$ \\
\hline \multirow{2}{*}{ 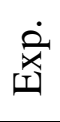 } & $d P[k P a]$ & 10.3 & 8.8 & 12.5 & 9.4 \\
\hline & $U\left[k W / m^{2} K\right]$ & 1.46 & 1.64 & 1.41 & 1.23 \\
\hline \multirow{2}{*}{$\dot{\Xi}$} & $d P[k P a]$ & 13.9 & 6.3 & 17.6 & 8.4 \\
\hline & $U\left[k W / m^{2} K\right]$ & 1.8 & 1.9 & 2.1 & 1.8 \\
\hline
\end{tabular}

Table 2: experimental and simulation results for the pressure drop and the heat transfer coefficients, obtained for the analyzed refrigerants and operating conditions.

\section{CONCLUSIONS}

The aim of this paper was to assess the applicability of the computational fluid dynamics, for the analysis of thermal behavior of a plate heat exchanger with refrigerant flow evaporation. Given the complexity of the occurring phase-change phenomena on the one hand, and on 
the other very demanding geometry which is considered, the focus here is on the robustness of the physical modeling and the stability of the numerical simulation.

Starting from the pseudo-fluid description of two-phase flows, the flow evaporation has been treated here in a single-phase flow manner. The transport properties of the pseudo-fluid have been defined as the weight mixture of the individual refrigerant properties (vapor and liquid), which are computed using CoolProp package. A standard transient OpenFoam solver has been extended to include the phase-change process, for which a robust physical description (based on a pre-defined function of the local temperature) has been deployed.

In order to validate the presented numerical approach, the comparison with the literature data has been performed. To that purpose, the geometry of the plate heat exchanger has been realistically represented, and the numerical analysis has been performed for the specified operating conditions. The simulation series has been performed for two different refrigerant and two operating conditions, and the results obtained from the numerical analysis compare reasonably well with the experimentally obtained values.

\section{ACKNOWLEDGEMENTS}

The financial support for the work presented in this paper has been provided by FFG Österreichische Forschungsförderungsgesellschaft mbH (project "HighButane", project number 843935).

\section{REFERENCES}

[1] I.H. Bell, J. Wronski, S. Quoilin, V. Lemort (2014). Pure and pseudo-pure fluid thermophysical property evaluation and the open-source thermophysical property library CoolProp. Industrial Engineering Chemistry Research 53:2498-2508.

[2] M. Colarossi, N. Trask, D.P. Schmidt, M.J. Bergander (2012). Multidimensional modeling of condensing two-phase ejector flow. International Journal of Refrigeration 35:290-299.

[3] H.G. Weller, G. Tabora, H. Jasak, C. Fureby (1998). A tensorial approach to computational continuum mechanics using object-oriented techniques. Computers in Physics 12:620-631.

[4] J. Huang, T.J. Sheer, M. Bailey-McEwan (2012). Heat transfer and pressure drop in plate heat exchanger refrigerant evaporators. International Journal of Refrigeration 35:325-335.

[5] C. Kleinstreuer (2003). Two-Phase Flow: Theory and Applications. Taylor \& Francis, New York, USA.

[6] W. Minkowycz (2009). Advances in numerical heat transfer. CRC Press, New York, USA.

[7] Jones, W. P. and Launder, B. E. (1972). The prediction of laminarization with a twoequation model of turbulence. Int. J. Heat Mass Transfer 15:301-314 